\title{
COLOR IMAGE WATERMARKING USING QUATERNION FOURIER TRANSFORM
}

\author{
Patrick Bas, Nicolas Le Bihan and Jean-Marc Chassery \\ Laboratoire des Images et des Signaux INPG/CNRS \\ 961 rue de la Houille Blanche Domaine universitaire \\ B.P. 4638402 Saint Martin d'Hères cedex FRANCE \\ Email: firstname.lastname@lis.inpg.fr
}

\begin{abstract}
This paper presents a digital color image watermarking scheme using the hypercomplex numbers representation and the Quaternion Fourier Transform (QFT). Previous color image watermarking methods are first presented and the quaternion representation is then described. In this framework RGB pixel values are associated with an unique quaternion number having three imaginary parts. The QFT is presented, this transform depends on an arbitrary unit pure quaternion $\boldsymbol{\mu}$. The value of $\boldsymbol{\mu}$ is selected to provide embedding spaces having robustness and/or perceptual properties. In the presented approach $\boldsymbol{\mu}$ is function of the mean color value of a block and a perceptual component. A watermarking scheme based on the QFT and the Quantization Index Modulation scheme is afterwards presented. This scheme is finally evaluated for different color image filtering process (JPEG, blur) and the fact that perceptive QFT embedding can offer robustness to luminance filtering techniques is outlined.
\end{abstract}

\section{INTRODUCTION}

Color images have not received as much attention from the watermarking community as grey-level images. One commonly used technique to address color images is to mark only the luminance component. If on one hand this solution is practical due to implementation issues, on the other hand the luminance component represents the most perceptive and sensible information. Consequently for watermarking and especially data-hiding methods, it is important not to discard chrominance information because such a channel represents an interesting amount of data that can be used for information embedding.

This paper is organised as follows: previous work on color image watermarking is first recalled. In the next section color images and color images transform are revisited using the hypercomplex representation and the Quaternion Fourier Transforms (QFT). In the next section a QFT is constructed

\footnotetext{
This work was supported by the french ARTUS RNRT project.
}

considering human perception issues. A watermarking scheme using this transform is afterwards presented and evaluated. Finally concluding remarks outline the different issues of this work.

\section{PREVIOUS WORK}

The aim of this section is to recall briefly important works on color image watermarking and data-hiding techniques.

$\diamond$ Kutter et. al. proposed a straightforward and practical solution: the blue channel is used as the embedding channel [1]. The authors claim that blue represents the less sensitive channel inside the RGB space. The embedding process uses spread spectrum techniques in the spatial domain.

$\diamond$ Piva et. al. proposed to take into account the three RVB components to increase the embedding space [2]. In this work, the power of the mark is based on the human eye sensibility to the light spectrum. The magnitude of the mark is consequently 10 times more significant for the blue component than for the green component and 5 times more significant for the blue component than for the red one. In the proposed scheme, the embedding process is an additive (spread-spectrum) embedding scheme in the DCT domain.

$\diamond$ Fleet et. al. used the Lab decomposition because such a decomposition presents interesting properties in the watermarking context [3]. The Lab space, representing an Euclidean sensitive space, can be extended to the S-CIELAB decomposition including spatial structure of the image. SCIELAB exploits the fact that color perception is different in homogeneous and in textured areas [4]. Signature embedding is performed adding a sinus pattern weighted by a mask coming from the Yellow/Blue S-CIELAB component. Signature detection is done finding local peak corresponding to the presence of the sinus.

\section{QUATERNION REPRESENTATION}

The use of quaternion number in color image processing is presented in this section. Quaternions also called hy- 
percomplex numbers has been introduced with color image representation by Sangwine and Ell [5][6]. A quaternion $q$ has four components, one real and three imaginary:

$$
q=w+x \boldsymbol{i}+y \boldsymbol{j}+z \boldsymbol{k}
$$

where $w, x, y$ and $z$ are real numbers, and $i, j$ and $\boldsymbol{k}$ are imaginary operators presenting the following properties:

$$
\begin{gathered}
i^{2}=j^{2}=k^{2}=i j k=-1 \\
i j=k \quad j k=i \quad k i=j \\
j i=-k \quad k j=-i \quad i k=-j
\end{gathered}
$$

Using this representation, a color image $I(x, y)$, sized by $N \times M$, can be considered as an array of pure quaternion numbers (e.g. with no real parts):

$$
I(x, y)=R(x, y) \boldsymbol{i}+G(x, y) \boldsymbol{j}+B(x, y) \boldsymbol{k}
$$

where $R(x, y), G(x, y)$ and $B(x, y)$ represent classical Red, Green and Blue color components. In [7] Sangwine and Ell have defined the quaternion Fourier (QFT) transform as:

$$
\operatorname{QFT}_{I}(v, u)=S \sum_{x=0}^{M-1} \sum_{y=0}^{N-1} e^{-\mu 2 \pi\left(\frac{x v}{M}+\frac{y u}{N}\right)} I(x, y)
$$

with the inverse transform defined by:

$$
I(x, y)=S \sum_{x=0}^{M-1} \sum_{y=0}^{N-1} e^{\mu 2 \pi\left(\frac{x v}{M}+\frac{y u}{N}\right)} Q F T_{I}(v, u)
$$

In these definitions, $S=1 / \sqrt{M N}$. An important point is that the QFT depends of the definition of $\boldsymbol{\mu}$. $\boldsymbol{\mu}$ has to represent any unit pure quaternion (e.g. $\mu^{2}=-1$ and $\Re(\boldsymbol{\mu})=0$ ). Such a transform can be easily computed using classical Fourier transforms and symplectic decomposition [8].

If the representation of the Fourier Transform of a signal can be easily interpreted using spectrum and phase information, in an analogue way the decomposition of the QFT was proposed in [7] to characterise two components parallel and orthogonal to $\boldsymbol{\mu}$ :

$$
Q F T_{I}(v, u)=Q F T_{\| I}(v, u)+Q F T_{\perp I}(v, u)
$$

$Q F T_{\| I}$ represents the component in which the signature will be embedded (cf section 5). The choice of $\boldsymbol{\mu}$ is explained in the next section.

\section{PERCEPTUAL QFT}

To design an efficient color watermarking scheme, we have to find a value of $\boldsymbol{\mu}$ such that the perceptual impact of the component $Q F T_{\| I}$ is minimal. Empirical tests were performed to find values of $\boldsymbol{\mu}$ that lead to minor visual distortion. Theses tests were accomplished adding "grating" on $8 \times 8$ blocks of natural images and evaluating the distortion on both CRT and LCD monitors. The value of $\boldsymbol{\mu}$ offering the smallest distortions was retained. Because $\boldsymbol{\mu}$ has to be unit and the QFT is an orthogonal transform, the quadratic error between the marked image $I_{W}$ and original image $I_{O}$ is equal to the quadratic error between the marked component $Q F T_{\| I_{W}}$ and the original component $Q F T_{\| I_{O}}$. Consequently the Mean Square Error between $I_{O}$ and $I_{W}$ is independent of the value of $\boldsymbol{\mu}$. These experiments yield to find different values of $\boldsymbol{\mu}$ providing small perceptual distortion. For example $\boldsymbol{\mu}_{\text {Perc }}=(-2 \boldsymbol{j}+8 \boldsymbol{k}) / \sqrt{68}$ permits the construction of a projection offering interesting masking properties for the different tested images. Figure 1 illustrates the perceptive impact of the perturbation of $Q F T_{\| I}$ with $\boldsymbol{\mu}=\boldsymbol{\mu}_{P e r c}$ and $\boldsymbol{\mu}=\boldsymbol{\mu}_{\text {Lum }}=(\boldsymbol{i}+\boldsymbol{j}+\boldsymbol{k}) / \sqrt{3}$ with same Mean Square Error (MSE) and Mean Absolute Error (MAE).

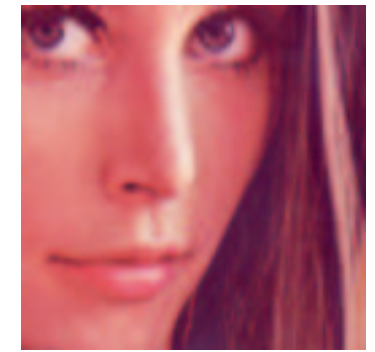

Part of $I_{O}$

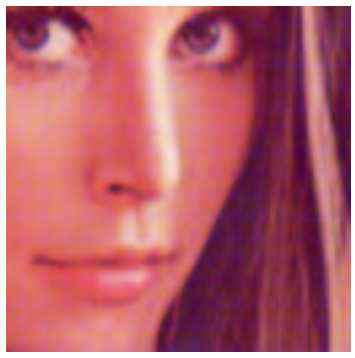

Part of $I_{W}\left(\boldsymbol{\mu}=\boldsymbol{\mu}_{\text {Perc }}\right)$

$\mathrm{MAE}=13.4$

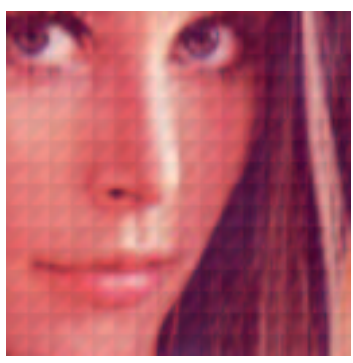

Part of $I_{W}\left(\boldsymbol{\mu}=\boldsymbol{\mu}_{L u m}\right)$

$\mathrm{MSE}=100, \mathrm{MAE}=13.4$

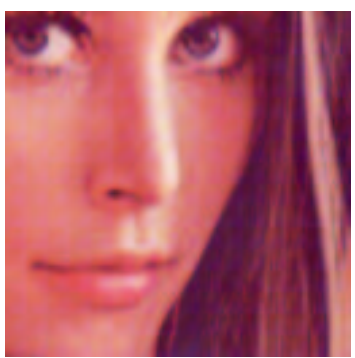

Part of $I_{W}\left(\boldsymbol{\mu}=\boldsymbol{\mu}_{\text {Perc }}\right)$

$\mathrm{MSE}=100$
Fig. 1. Effect of grating insertion according to different values of $\boldsymbol{\mu}$.

Even if the previous choice $\boldsymbol{\mu}=\boldsymbol{\mu}_{P e r c}$ yields to a satisfactory perceptual embedding domain, we have observed that the masking effect was not independent of the mean color of the block. For example for $\boldsymbol{\mu}_{\text {Perc }}=(-2 \boldsymbol{j}+$ $8 \boldsymbol{k}) / \sqrt{68}$, the masking effect is less significant in blue areas that in other colored areas. Consequently we decide to find 
$\boldsymbol{\mu}$ as function of $\boldsymbol{\mu}_{I m}$ and $\boldsymbol{\mu}_{P e r c} \cdot \boldsymbol{\mu}_{I m}$ represents an unit pure quaternion parallel to the image color component:

$$
\boldsymbol{\mu}_{I m}=\frac{E_{b}[R] \boldsymbol{i}+E_{b}[G] \boldsymbol{j}+E_{b}[B] \boldsymbol{k}}{\sqrt{E_{b}^{2}[R]+E_{b}^{2}[G]+E_{b}^{2}[B]}}
$$

where $E_{b}[X]$ represents the mean of the component $X$ in a block $b$ of the image.

We have found that $\boldsymbol{\mu}_{\boldsymbol{O} \boldsymbol{r} \boldsymbol{t h}}$ representing the quaternion perpendicular to $\boldsymbol{\mu}_{I m}$ and belonging to the plan $\mathcal{P}_{P \text { erc }}$ that includes $\boldsymbol{\mu}_{I m}$ and $\boldsymbol{\mu}_{P e r c}$ is a good candidate:

$$
V\left(\boldsymbol{\mu}_{\text {Orth }}\right)=V\left(\boldsymbol{\mu}_{\text {Im }}\right) \wedge\left[V\left(\boldsymbol{\mu}_{\text {Im }}\right) \wedge V\left(\boldsymbol{\mu}_{\text {Perc }}\right)\right]
$$

Here $V(q)$ denotes the 3D vector corresponding to the imaginary part of $q: V(w+x \boldsymbol{i}+y \boldsymbol{j}+z \boldsymbol{k})=\left[\begin{array}{ll}x & y \\ z\end{array}\right]^{T}$. The product $\wedge$ is the cross-product. The geometrical interpretation of this operation is illustrated on figure 2. Masking effect can be improved rotating $\mu_{\text {Orth }}$ by an angle $\theta$. We obtain the quaternion $\boldsymbol{\mu}_{\boldsymbol{P e r c ^ { \prime }}}$ in the plan $\mathcal{P}_{\boldsymbol{P e r c}}$ :

$$
V\left(\boldsymbol{\mu}_{\boldsymbol{P e r c ^ { \prime }}}\right)=\mathcal{R}_{\mathcal{P}_{\text {Perc }}}\left(V\left(\boldsymbol{\mu}_{\text {Perc }}\right), \theta\right)
$$

where $\mathcal{R}_{\mathcal{P}}\left(V_{1}, \theta\right)$ means the rotation of $V_{1}$ with an angle $\theta$ in the plan $\mathcal{P}$. $\theta=\pi / 10$ leads to very satisfactory masking properties.

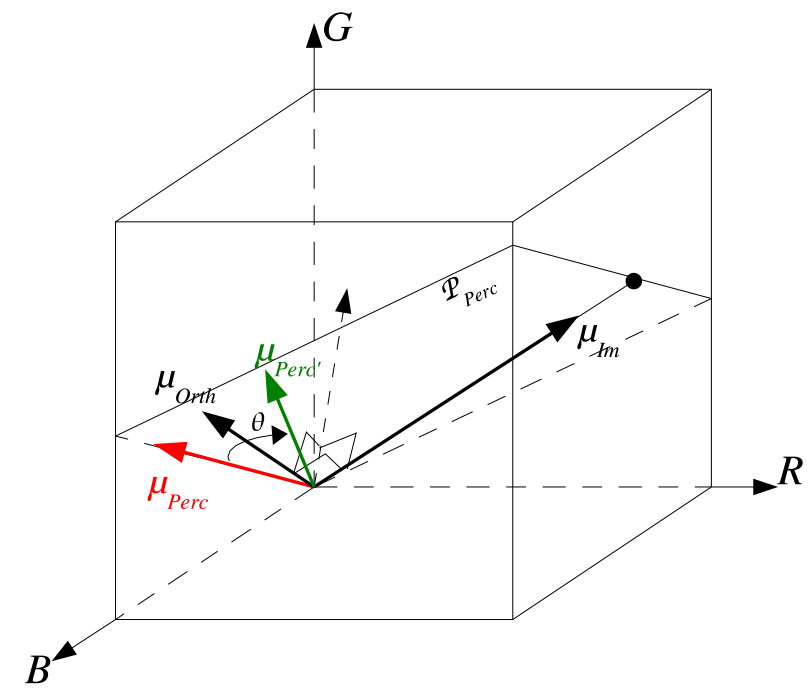

Fig. 2. Construction of $\mu_{\text {Orth }}$ and $\mu_{P e r c^{\prime}}$.

\section{EMBEDDING AND DETECTION SCHEME}

To evaluate and assess the interest of using the perceptive QFT transform we have decided to embed a mark into $Q F T_{\| I}$. This transform is applied on a $8 \times 8$ block of the original image. We choose a classical watermarking and data-hiding scheme named Quantization Index Modulation scheme proposed by [9]. Such an approach can achieve both capacity and robustness requirements. Consequently the embedding of the bitstream $b[n]$ is performed by quantizing the coefficient $C=\Re\left(Q F T_{\| I}(1,1)\right)$ using the following algorithm:

$$
\begin{array}{ll}
\text { Coding step: } & \\
\text { If } \quad b[i]=1: & C_{w}=2 \Delta \text { Round }\left(\frac{C}{2 \Delta}\right)+\Delta / 2 \\
\text { If } \quad b[i]=0: & C_{w}=2 \Delta \operatorname{Round}\left(\frac{C}{2 \Delta}\right)-\Delta / 2
\end{array}
$$

The decoding of the signature if performed using the following algorithm:

$$
\begin{aligned}
& \text { Decoding step: } \\
& \text { If } \\
& C-2 \Delta \text { Round }\left(\frac{C}{2 \Delta}\right)>0 \\
& \Rightarrow b[i]=1 \\
& \text { Else } \\
& \Rightarrow b[i]=0
\end{aligned}
$$

\section{RESULTS}

The goal of this section is to argue the different advantages offered by the presented scheme in term of visual impact and robustness to distortions. The robustness was evaluated using the JPEG compression technique and a Gaussian blurring filter. The bit error rate (BER) has been evaluated using either $\boldsymbol{\mu}=\boldsymbol{\mu}_{\text {Perc }}$ or $\boldsymbol{\mu}=\boldsymbol{\mu}_{\text {Lum }}$ with equal Mean Absolute Error. The MAE was used because the $L^{1}$ norm produces values that are more conform to visual error than the $L^{2}$ norm (MSE). The different results are presented on Table 1 and Table 2.

These results induce two important remarks. On one hand, results presented on Table 1 illustrate the property that our perceptive embedding scheme is more robust to luminance filtering techniques than the luminance embedding scheme. This property is due to the fact that ${ }_{Q F T} F I(v, u)$ is orthogonal to the luminance axis. On the other hand Table 2 outlines the fact that the perceptive embedding scheme is less robust to JPEG compression than the luminance embedding scheme. Such a result is explained by the fact that the JPEG compression scheme can dissociate the Luminance and the Chrominance components and that the DCT quantization matrix can be distinct for Luminance and Chrominance coefficients (the quantization step is more important for chroma coefficients). Moreover, in the JPEG compression technique, the chroma components can be sub-sampled. Consequently the component ${ }_{2 F T_{\| I}}(v, u)$ is more damaged for $\boldsymbol{\mu}=\boldsymbol{\mu}_{\text {Perc }}$ than for $\boldsymbol{\mu}=\boldsymbol{\mu}_{\text {Lum }}$. Nevertheless as it is shown in Figure 3, for a similar BER, the visual impact of the perceptual embedding scheme is less disturbing than the visual impact of the luminance embedding scheme.

\section{CONCLUDING REMARKS}

In this paper a new watermarking/data-hiding technique for digital color images has been presented. The Quaternion 


\begin{tabular}{|c|c|c|c|}
\hline MAE & 5 & 10 & 20 \\
\hline $\boldsymbol{\mu}=\boldsymbol{\mu}_{\text {Perc }}: \operatorname{BER}(\%)$ & 10.44 & 4.73 & 1.09 \\
\hline $\boldsymbol{\mu}=\boldsymbol{\mu}_{\text {Lum }}: \operatorname{BER}(\%)$ & 29.54 & 20.04 & 13.45 \\
\hline
\end{tabular}

Table 1. BER results for gaussian blurring.

\begin{tabular}{|c|c|c|c|}
\hline MAE & 5 & 10 & 20 \\
\hline $\boldsymbol{\mu}=\boldsymbol{\mu}_{\text {Perc }^{\prime}}: \mathrm{BER}(\%)$ & 50 & 4.46 & 0.12 \\
\hline $\boldsymbol{\mu}=\boldsymbol{\mu}_{\text {Lum }}: \mathrm{BER}(\%)$ & 0.26 & 0.0 & 0.0 \\
\hline
\end{tabular}

Table 2. BER results for JPEG compression (Quality factor $=85 \%$ ).

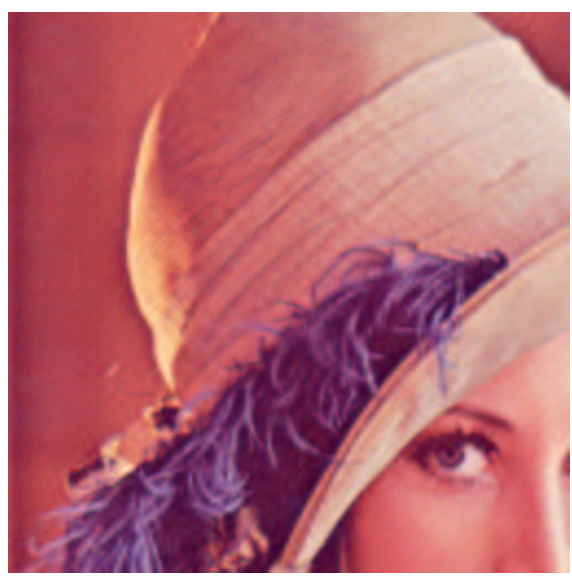

Part of $I_{W}\left(\boldsymbol{\mu}=\boldsymbol{\mu}_{L u m}\right)$

$\mathrm{JPEG}=85 \%, \mathrm{BER}=0.4 \%, \mathrm{MAE}=4.6$

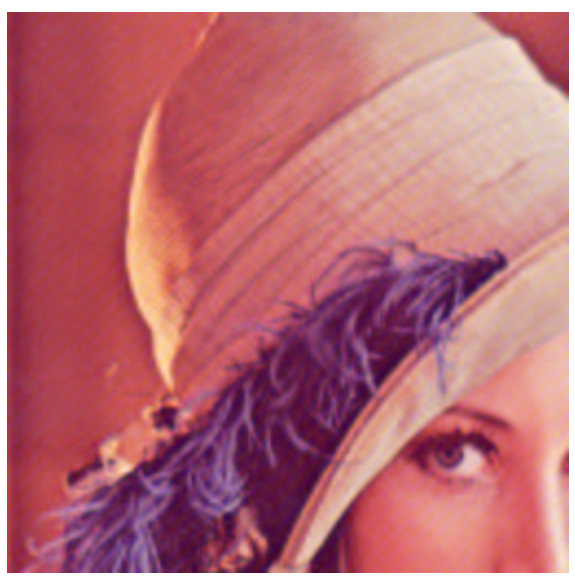

Part of $I_{W}\left(\boldsymbol{\mu}=\boldsymbol{\mu}_{P e r c^{\prime}}\right)$

$\mathrm{JPEG}=85 \%, \mathrm{BER}=0.4 \%, \mathrm{MAE}=13.6$

Fig. 3. Evaluation of the visual impact of the embedding process on marked images for similar robustness after JPEG compression with a quality factor of $85 \%$.

representation and the Quaternion Fourier Transform were used because a pixel value is processed without dissociating the different color. The construction of the QFT is associated with the choice of an unit pure quaternion $\boldsymbol{\mu}$ that can be selected to induces an embedding space which is not perceptually affected by the signature embedding. Moreover the QFT has permitted the design of a robust watermarking scheme in the frequential domain. The proposed perceptual scheme is not robust to JPEG compression and is more suited for data-hiding applications. Future work will be devoted to embed signature both in perceptual and luminance components to provide similar robustness for luminance filtering and compression techniques.

\section{REFERENCES}

[1] M. Kutter, F. Jordan, and F. Bossen, "Digital signatures of color images using amplitude modulation," in SPIE, EI97 Proceedings, San Jose, California USA, Feb. 1997, pp. 518-526.

[2] A. Piva, M. Barni, F. Bartolini, and V. Cappellini, "Exploiting the cross-correlation of rgb-channels for robust watermarking of color images," in IEEE-ICIP'99, Kobe (Japan), Oct. 1999, vol. I, pp. 306-310.

[3] D. Fleet and D. Heeger, "Embedding invisible information in color images," in IEEE-ICIP'97, Santa Barbara (Cal) Usa, 1997, vol. 1, pp. 532-535.

[4] X. M. Zhang and B. A. Wandell, "A spatial extension to cielab for digital color image reproduction," Society of Information Display Sumposium Technical Digest, vol. 27, pp. 731-734, 1996.

[5] C.J. Evans, S. Sangwine, and T. Ell, "Colour-sensitive edge detection using hypercomplex filters," in Proc. EUSIPCO, Tampere, Finland, Sept 2000, vol. I, pp. 107-110.

[6] C.J. Evans, S. Sangwine, and T. Ell, "Hypercomplex color-sensitive smoothing filters," in Proc. ICIP, Sept 2000, vol. 1, pp. 541-544.

[7] S. Sangwine and T. Ell, "Hypercomplex fourier transforms of color images," in Proc. ICIP, Thessaloniki, Greece, 2001, vol. 1, pp. 137-140.

[8] T. Ell and S. Sangwine, "Decomposition of 2d hypercomplex fourier transforms into pairs of complex fourier transforms," in Proc. EUSIPCO, Tampere, Finland, Sept 2000, vol. II, pp. 1061-1064.

[9] B. Chen and G. W. Wornell, "Quantization index modulation: A class of provably good methods for digital watermarking and information embedding," IEEE Transactions on Information Theory, vol. 47, no. 4, pp. 1423-1443, 2001. 